\title{
26463 - BLOOD TRANSFUSION IN LIVE AND DECEASED DONOR LIVER
}

TRANSPLANTATION

\section{Huan Zhang MD, Stuart McCluskey, MD PhD; Keyvan Karkouti, MD; David Grant, David Bevan, MB; Toronto General Hospital, Toronto, ONTARIO, Canada}

INTRODUCTION: Compared with traditional deceased donor (DD) orthotopic liver transplantation (OLT), live donor (LD) OLT is purported to have many benefits, one of which is reduced perioperative red blood cell (RBC) transfusion requirements. This would be an important advantage given that RBC transfusions have been shown to increase morbidity and mortality in a dose-dependent manner. In this observational study, we sought to determine if, compared with DD-LDT, RBC transfusion rates were lower in LD-OLT.

METHODS: Following REB approval, the charts of patients undergoing OLT between January 2001 and December 2005 at a single academic institution were reviewed for patient demographics, etiology of liver failure, co-morbidities (coronary artery disease, pulmonary disease, ascites, encephalopathy, varices, and renal failure), preoperative laboratory results (hemoglobin concentration, platelet count, creatinine, albumin, bilirubin, International Normalized Ratio), previous abdominal surgery, anesthetic management, transfusion requirements within the first 24 hour from the start of transplantation (primary outcome), and postoperative adverse events. Patients with fulminant liver failure or undergoing repeat transplantation during the same hospital stay as the original transplantation were excluded. LD and DD-OLT patients were compared for continuous (t-test or Mann-Whitney U test) and categorical (chi-square or Fisher's exact test) variables.

RESULTS: A total of 313 patients were included in this study. Patients undergoing LDOLT $(n=96)$ were similar in the age, gender, height and weight to those of patients undergoing DD-OLT $(n=217)$. Viral hepatitis $(51 \%$ vs.40\%: $\mathrm{p}=0.049)$ and PSC $(13 \%$ vs.6\%: $\mathrm{p}=0.028$ ) were more common in LD-OLT than DD-OLT. No significant difference was found for preoperative laboratory results between groups. Apart from hepatorenal syndrome that was more common in DD-OLT than LD-OLT ( $12 \%$ vs. $5 \%$; $p=0.049)$, there was no difference in the incidence of co-morbidities. No differences were found in the number of patients that required no $\operatorname{PRBC}(\mathrm{p}=0.726)$, massive transfusion $(\mathrm{p}=0.550)$ or the number of units of PRBC transfused per patient ( $\mathrm{p}=0.648)$ (Table 1). In addition the transfusion requirements of other blood products, fresh frozen plasma, platelets and cryoprecipitate, were the same between groups.

CONCLUSIONS: After excluding patients with fulminant liver failure there was no difference between the patient populations undergoing LD or DD OLT at our institution, which would influence blood loss. The transfusion requirements of OLT are same between these 2 groups. 
Table 1: Blood Transfusion Requirements in the First 24hr of Liver Transplantation

\begin{tabular}{|l|c|c|c|}
\hline & No RBC & $\begin{array}{c}\text { Massive Transfision } \\
\text { ( } 6 \text { Units RBC) }\end{array}$ & $\begin{array}{c}\text { \# of Units/Patient } \\
\text { Median } \\
\text { (interquartile range) }\end{array}$ \\
\hline LDLT (n-96) & $15(16 \%)$ & $37(38 \%)$ & $4(2,7)$ \\
\hline $\begin{array}{l}\text { CDLT } \\
(\mathrm{n}=217)\end{array}$ & $35(17 \%)$ & $76(35 \%)$ & $4(2,7)$ \\
\hline
\end{tabular}

